\section{Conhecimento e uso de mecanismos para articulação clínica entre níveis em duas redes de atenção à saúde de Pernambuco, Brasil}

\author{
Awareness and use of mechanism for clinical \\ coordination between levels in two health care \\ networks in Pernambuco State, Brasil
}

\section{Conocimiento y uso de mecanismos para la coordinación clínica entre niveles de asistencia en dos redes de atención de salud en Pernambuco, Brasil}

\author{
Cecylia Roberta Ferreira de Oliveira 1 \\ Isabella Chagas Samico 1 \\ Marina Ferreira de Medeiros Mendes 1 \\ Ingrid Vargas 2 \\ Maria Luisa Vázquez ${ }^{2}$
}

\section{Resumo}

$O$ artigo avalia o conhecimento e o uso dos mecanismos de articulação clinica entre níveis assistenciais em duas redes de atenção à saúde no Estado de Pernambuco, Brasil. Trata-se de estudo descritivo e transversal, do tipo inquérito. Foram entrevistados 381 médicos das redes públicas de saúde da atenção básica e atenção especializada dos municípios de Caruaru e Recife (Distritos Sanitários III e VII). Foi aplicado um questionário estruturado (COORDENA) para avaliar o conhecimento, frequência e características no uso dos seguintes mecanismos: formulário de referência e contrarreferência, resumo de alta, telefone e bilhetes (mecanismos de adaptação mútua), protocolos do Ministério da Saúde e sessões clínicas conjuntas (mecanismos de padronização). Os dados foram analisados por meio de frequências simples, médias e percentuais. De modo geral, os médicos da atenção básica conhecem e utilizam mais os mecanismos que os médicos da atenção especializada. $\mathrm{Na}$ comparação entre redes, Recife apresentou os melhores resultados. Os formulários de referência e contrarreferência foram os mais usados $(61,4 \%)$ $e$ as sessões clinicas conjuntas as de menor uso (8,6\%), além da existência de mecanismos informais (telefone 58\%, bilhete 56,6\%, WhatsApp 2,6\%). A subutilização dos mecanismos, a divergência de informações no envio e recebimento entre os profissionais da atenação básica e atenção especializada, e o uso inadequado dos mesmos sugerem falhas na comunicação entre os profissionais e entre os níveis de atenção. Os achados revelam a necessidade de investimentos que propiciem o conhecimento, a comunicação e a colaboração entre profissionais, contribuindo para uma melhor articulação entre os diferentes niveis assistenciais.

Níveis de Atenção à Saúde; Assistência Integral à Saúde; Integralidade em Saúde; Avaliação em Saúde

\author{
Correspondência \\ I. C. Samico \\ Instituto de Medicina Integral Prof. Fernando Figueira. \\ Rua dos Coelhos 300, Recife, PE 50070-550, Brasil. \\ isabella@imip.org.br \\ 1 Instituto de Medicina Integral Prof. Fernando Figueira, Recife, \\ Brasil. \\ 2 Consorci de Salut i Social de Catalunya, Barcelona, España.
}




\section{Introdução}

No Brasil, o Sistema Único de Saúde (SUS) apresenta vários problemas no cumprimento de seus princípios, diretrizes e objetivos, mesmo com os avanços que evidenciaram o seu fortalecimento. Questões relacionadas à coordenação dos cuidados em saúde, fragmentação da rede assistencial e ausência de comunicação entre níveis são temas recorrentes nas discussões sobre a organização do sistema de saúde 1 .

As mudanças no perfil epidemiológico, demográfico e nutricional da população brasileira evidenciam falhas e o desencontro entre a prevalência das doenças crônicas não transmissíveis (DCNT) e um sistema de saúde voltado para responder às condições agudas 2,3,4. Em 2012, as DCNT foram responsáveis por 67,8\% (38 milhões) de todas as mortes que ocorreram no mundo e, destas, $75 \%$ ocorreram em países de média e baixa rendas. O Brasil acompanha essa tendência mundial, onde $74 \%$ das mortes no país foram devido às DCNT 5 .

Com a finalidade de promover uma atenção em saúde mais eficiente, integral e de maior qualidade, foram instituídas as Redes Integradas de Serviços de Saúde (RISS) em diversos sistemas de saúde, como é o caso da América Latina 2,3. No Brasil, as Redes de Atenção à Saúde (RAS) foram criadas pelo Ministério da Saúde por meio da Portaria no 4.279, em 2010, e pelo Decreto no 7.508, em 2011 2,6. As RAS são organizações poliárquicas que promovem a igualdade entre os serviços de saúde que têm os mesmos objetivos e almejam uma atenção contínua nos três níveis assistenciais, sob coordenação da atenção básica 3 .

Na RAS, a falta de coordenação assistencial é um dos principais obstáculos a serem superados para promover a articulação entre níveis 1,3,7. A coordenação assistencial define-se como um acordo de todos os serviços relacionados com a atenção do paciente, com independência do lugar onde se oferte, de maneira que se harmonizem e alcancem um objetivo comum, administrando os possíveis conflitos e centrada na interação entre provedores 8 .

Para a melhoria da coordenação é necessário minimizar as barreiras entre níveis assistenciais, promovendo um "sistema sem muros", por meio da implantação de mecanismos e estratégias de articulação entre níveis 8,9,10. Os mecanismos objetivam facilitar a transferência de informações, aperfeiçoar a troca de conhecimentos e melhorar a comunicação dos profissionais, contribuindo para a continuidade do cuidado e a coordenação da atenção ${ }^{9}$. Os mecanismos podem ser de padronização - específicos do processo, resultado ou habilidades (sessões clínicas e protocolos compartilhados) ou de adaptação mútua - maior contato entre participantes envolvidos no processo de trabalho em situações de difícil planejamento (formulário de referência e contrarreferência, resumo de alta hospitalar, e-mail, telefone e bilhete) $3,8,10$.

No Brasil, várias políticas auxiliam na implementação dos mecanismos. O sistema de referência e contrarreferência, por exemplo, é considerado como um dos elementos-chave para a efetividade das RAS, e o Ministério da Saúde ressaltou o papel deste mecanismo e dos protocolos compartilhados para a integração entre os níveis de atenção e o aumento da resolubilidade da atenção básica 3,11,12. A implementação dos mecanismos está relacionada com o sistema de saúde e com fatores políticos, econômicos e organizacionais. Por isso, a gestão deve incentivar o uso e reforçar estratégias para a utilização na conduta clínica 11,12. Porém, estudos sobre mecanismos que promovam a coordenação e continuidade da atenção entre níveis ainda são escassos e as evidências sobre este tema são limitadas, principalmente na América Latina 11,12,13,14.

O presente artigo faz parte de uma pesquisa denominada Impacto de Estratégias de Integração da Atenção no Desempenho das Redes de Serviços de Saúde em Diferentes Sistemas de Saúde da América Latina (EQUITY-LA II), que avalia a efetividade de diferentes estratégias de integração da atenção na melhoria da coordenação e da qualidade da atenção nas RAS da América Latina 12.

Para este artigo, o objetivo consistiu em avaliar o conhecimento e o uso dos mecanismos de articulação entre níveis assistenciais em duas redes de atenção à saúde no Estado de Pernambuco, Brasil. 


\section{Métodos}

Trata-se de um estudo descritivo e transversal, do tipo inquérito, realizado em dois municípios pernambucanos: Recife (Distrito Sanitário - DS III e VII), capital do estado, e Caruaru, maior cidade do agreste pernambucano. As redes dos DS III e VII são contínuas e têm 32 unidades de atenção básica e 14 centros de atenção especializada para atender a 312.981 habitantes. Caruaru tem uma população de 351.686 habitantes e sua rede assistencial é formada por 63 unidades de atenção básica e 19 centros de atenção especializada (Departamento de Atenção Básica, Ministério da Saúde. Cobertura da Estratégia Saúde da Família, 2016. http://dab.saude.gov.br/portaldab/historico_cobertura_sf.php, acessado em 20/Ago/2016). Tais redes foram selecionadas baseando-se nos seguintes critérios: ter uma rede de serviços contínua, incluindo a atenção básica e atenção especializada; ter serviços com área e população delimitada; contemplar áreas de médio/baixo nível socioeconômico; e contar com profissionais que aceitassem participar da pesquisa 12.

A população do estudo foi constituída por médicos da atenção básica e da atenção especializada que atuavam há mais de três meses na rede assistencial. O tamanho da amostra foi de 174 médicos por rede (com um mínimo de 50 médicos da atenção básica) e calculada com a finalidade de assegurar a detecção de uma variação de $15 \%$ entre redes no uso dos mecanismos de coordenação clínica e na percepção dos médicos/as sobre a coordenação da atenção entre níveis nas redes. Para tanto, adotou-se um poder de $80 \%(\beta=0,20)$ e um nível de $95 \%$ de confiança $(\alpha=0,05)$. Para atingir a amostra, todos os médicos das redes (que cumpriam os critérios de seleção) foram convidados a participar da pesquisa, com um percentual de recusa de 6,6\% na rede de Caruaru e 13,4\% em Recife (DS III e VII) 12.

Os dados foram coletados por meio de entrevistas realizadas por pesquisadores capacitados, no período de junho a setembro de 2015, utilizando-se um questionário estruturado (COORDENA) 12. Esse questionário foi traduzido, adaptado e validado pela equipe de pesquisa. Em Caruaru, o estudo ocorreu em 41 unidades de saúde da família (USF) da zona urbana, 12 da zona rural e 16 centros de atenção especializada. Em Recife (DS III e VII), as entrevistas foram realizadas em 22 unidades de atenção básica e em 14 centros de atenção especializada.

A supervisão direta das entrevistas no campo, a revisão de todos os questionários e a repetição de entrevistas com $20 \%$ dos participantes selecionados aleatoriamente foram estratégias utilizadas para garantir a qualidade das respostas e a confiabilidade da pesquisa. As inconsistências encontradas foram corrigidas pela checagem do banco de dados e pela dupla entrada das respostas nos bancos.

Consideraram-se as variáveis sociodemográficas (sexo, idade e nacionalidade), laborais (nível de atenção, especialidade e experiência no local de trabalho), relacionadas com o conhecimento dos mecanismos de articulação entre níveis (existência, finalidade), com a utilização desses mecanismos (frequência de envio e recebimento e de utilização, características do uso, conteúdo, dificuldades) e variáveis organizacionais (disponibilidade de tempo para a articulação clínica). A finalidade do uso e as dificuldades foram identificadas por perguntas abertas presentes no questionário, que posteriormente foram codificadas e analisadas.

As variáveis selecionadas foram avaliadas por nível assistencial e por rede de atenção. Algumas variáveis de interesse foram dicotomizadas para uma melhor comparação entre níveis e redes: frequência de envio e recebimento do formulário de referência, formulário de contrarreferência, resumo de alta hospitalar, seguimento dos protocolos, de participação das sessões clínicas e de utilização do telefone e bilhetes, recebimento do formulário de referência e formulário de contrarreferência em tempo útil e tempo de consulta disponível para a articulação clínica. Para a dicotomização, as respostas "sempre, muitas vezes" foram consideradas como alta frequência na utilização e "às vezes, muito poucas vezes e nunca" como baixa frequência. Em relação aos bilhetes, "diariamente e semanalmente" foram consideradas alta frequência; "mensalmente e com menor frequência" foram categorizadas como baixa frequência de uso 13 .

Os dados foram analisados por meio de frequências simples, percentuais e médias, apresentados em tabelas e gráficos. Foram processados no programa SPSS, versão 23.0 (https://www.ibm.com/) e pelo Microsoft Excel 2010 (https://products.office.com/).

O presente estudo segue os princípios éticos, segundo a Resolução no 466/2012 do Conselho Nacional de Saúde, que regulamenta as pesquisas envolvendo seres humanos, e foi aprovado pelo Comitê 
de Ética em Pesquisa em Seres Humanos do Instituto de Medicina Integral Prof. Fernando Figueira (IMIP) com o parecer no 1.995.731 e CAAE: 164439017.0.0000.5201.

\section{Resultados}

Dos 381 médicos entrevistados nas duas redes, a maioria era do sexo feminino $(67,7 \%)$ na rede Recife, e um pouco mais da metade do sexo masculino na rede Caruaru (53,3\%). A maioria tem nacionalidade brasileira, mas ressalta-se o papel do Programa Mais Médicos e do Programa de Valorização do Profissional da Atenção Básica (PROVAB), com a presença de médicos cubanos compondo mais de $10 \%$ da amostra total, especialmente na atenção básica (32,8\%) na rede Caruaru. Em torno de $40 \%$ dos participantes estavam na faixa etária de 50 anos ou mais na rede Recife. Entretanto, na rede Caruaru verificou-se maior prevalência de profissionais mais jovens. A maior parte dos entrevistados de ambas as redes possuía tempo de experiência na rede de mais de 36 meses (60,7\% rede Recife e $41,4 \%$ rede Caruaru) e vínculo institucional público estatutário (69,2\% rede Recife e 59,4\% rede Caruaru), à exceção dos médicos da atenção básica na rede Caruaru (Tabela 1).

Quanto ao conhecimento da existência dos mecanismos de articulação entre níveis nas redes estudadas, em Caruaru o telefone institucional foi o mais reconhecido (59,4\%), seguido do resumo de alta hospitalar $(48,9 \%)$, do formulário de referência e contrarreferência $(48,3 \%)$, de protocolos do Ministério da Saúde (31,7\%) e das sessões clínicas conjuntas (6,7\%). Diferentemente, na rede Recife, o formulário de referência e formulário de contrarreferência foram os mecanismos mais reconhecidos $(73,1 \%)$, seguidos pelo resumo de alta hospitalar $(60,2 \%)$, telefone institucional $(56,7 \%)$, protocolos do Ministério da Saúde (34,3\%) e das sessões clínicas conjuntas (10,4\%) (Tabela 2).

Quando questionados sobre a finalidade de uso dos formulários de referência e contrarreferência, a maioria dos médicos nas duas redes (75\% em Caruaru e 70,2\% em Recife) considerou a troca de informações entre níveis assistenciais, apesar do encaminhamento de pacientes e da necessidade burocrática também terem sido citados como motivos de uso. A principal finalidade no uso do resumo de alta hospitalar, em Caruaru e em Recife, era enviar/receber informações para outro nível (87,5\% e $85,1 \%$, respectivamente) e dos protocolos do Ministério da Saúde foi o mesmo nas duas redes: padronizar a atenção e o acompanhamento ao paciente, com 36,8\% na rede Caruaru e 51,1\% na rede Recife (Tabela 2).

Em relação aos bilhetes, $49 \%$ dos médicos de Caruaru e 44,1\% dos profissionais em Recife relataram como a principal finalidade a troca de informações entre níveis. Dentre os poucos médicos que responderam que participavam das sessões clínicas conjuntas, aprimorar o conhecimento era a principal função para 58,3\% dos médicos de Caruaru e 52,4\% de Recife (Tabela 2).

Quando questionados sobre a utilização de telefones (institucional e pessoal) para tratar temas referentes ao atendimento de pacientes, o telefone pessoal era mais utilizado que o institucional nas duas redes (35,6\% em Caruaru e 46,3\% em Recife). O bilhete era amplamente usado em ambas as redes, com maior participação dos especialistas sob a justificativa de trocar informações entre níveis (40,7\% na atenção básica e 45,1\% na atenção especializada). Dentre aqueles que usavam os protocolos, a frequência de uso era alta na atenção básica e na atenção especilaizada nas duas redes de estudo. Na rede Caruaru, as sessões clínicas e os bilhetes tinham maior participação dos profissionais da atenção básica do que da atenção especilaizada, diferentemente de Recife, com frequência de participação semelhante nos dois níveis de atenção.

Quanto ao envio e recebimento dos formulário de referência contrarreferência, houve uma discrepância entre os níveis de atenção, principalmente quanto ao formulário de contrarreferência que a maioria dos especialistas afirmava enviar e poucos médicos da atenção básica citavam receber. Essa diferença também ocorreu quanto ao envio e recebimento do resumo de alta hospitalar, mas com uma menor diferença quando comparado ao formulário de referência e formulário de conrarreferência (Figura 1).

A frequência no seguimento dos protocolos do Ministério da Saúde compartilhados entre níveis mostrou-se alta para as duas redes e para os dois níveis de atenção, diferentemente das sessões clínicas, com uma alta frequência por parte da atenção básica (rede Caruaru). Verificou-se uma alta frequência de uso do telefone por cerca de $40 \%$ dos profissionais nos dois níveis e nas duas redes, e 
Tabela 1

Características sociodemográficas e laborais da amostra. Caruaru e Recife (DS III e VII), Pernambuco, Brasil, 2015.

\begin{tabular}{|c|c|c|c|c|c|c|}
\hline \multirow[t]{3}{*}{ Características } & \multicolumn{3}{|c|}{ Caruaru } & \multicolumn{3}{|c|}{ Recife } \\
\hline & $\begin{array}{l}\text { Atenção } \\
\text { básica } \\
(n=58)\end{array}$ & $\begin{array}{c}\text { Atenção } \\
\text { especializada } \\
(n=122)\end{array}$ & $\begin{array}{c}\text { Total } \\
(\mathrm{N}=180)\end{array}$ & $\begin{array}{l}\text { Atenção } \\
\text { básica } \\
(n=51)\end{array}$ & $\begin{array}{c}\text { Atenção } \\
\text { especializada } \\
(n=150)\end{array}$ & $\begin{array}{c}\text { Total } \\
(N=201)\end{array}$ \\
\hline & n (\%) & n (\%) & n (\%) & n (\%) & n (\%) & n (\%) \\
\hline \multicolumn{7}{|l|}{ Sexo } \\
\hline Feminino & $34(58,6)$ & $50(41,0)$ & $84(46,7)$ & $34(66,7)$ & $102(68,0)$ & $136(67,7)$ \\
\hline Masculino & $24(41,1)$ & $72(59,0)$ & $96(53,3)$ & $17(33,3)$ & $48(32,0)$ & $65(32,3)$ \\
\hline \multicolumn{7}{|l|}{ Nacionalidade } \\
\hline Brasil & $38(65,5)$ & $120(98,4)$ & $158(87,8)$ & $46(90,2)$ & $150(100,0)$ & $196(97,5)$ \\
\hline Cuba & $19(32,8)$ & $1(0,8)$ & $20(11,1)$ & $3(5,9)$ & $0(0,0)$ & $3(1,5)$ \\
\hline Outras (Paraguai, Bolívia) & $1(1,7)$ & $1(0,8)$ & $2(1,1)$ & $2(3,9)$ & $0(0,0)$ & $2(1,0)$ \\
\hline \multicolumn{7}{|l|}{ Faixa etária (anos) } \\
\hline $24-34$ & $20(34,5)$ & $31(25,4)$ & $51(28,3)$ & $13(26,0)$ & $34(23,0)$ & $47(23,7)$ \\
\hline $35-49$ & $31(53,4)$ & $56(45,9)$ & $87(48,3)$ & $12(24,0)$ & $58(39,2)$ & $70(35,4)$ \\
\hline $50-76$ & $7(12,1)$ & $35(28,7)$ & $42(23,3)$ & $25(50,0)$ & $56(37,8)$ & $81(40,2)$ \\
\hline \multicolumn{7}{|l|}{$\begin{array}{l}\text { Especialização médica (atenação básica/ } \\
\text { atenção especializada) }\end{array}$} \\
\hline Clínica geral & $30(51,7)$ & $19(15,6)$ & $49(27,2)$ & $14(27,4)$ & $7(4,6)$ & $21(10,4)$ \\
\hline Pediatria & $2(3,4)$ & $21(17,2)$ & $23(12,8)$ & $5(9,8)$ & $39(26,0)$ & $44(21,9)$ \\
\hline Ginecologia/Obstetrícia & $0(0,0)$ & $17(13,9)$ & $17(9,4)$ & $0(0,0)$ & $19(12,7)$ & $19(9,5)$ \\
\hline Medicina da família/Comunidade & $12(20,7)$ & $2(1,6)$ & $14(7,8)$ & $20(39,2)$ & $0(0,0)$ & $20(10,0)$ \\
\hline Outras & $14(24,2)$ & $63(51,7)$ & $77(42,8)$ & $12(23,6)$ & $85(56,7)$ & $97(48,2)$ \\
\hline \multicolumn{7}{|l|}{ Tempo de experiência na rede (meses) } \\
\hline$\leq 12$ & $21(36,2)$ & $18(14,8)$ & $39(21,7)$ & $12(23,5)$ & $22(14,7)$ & $34(16,9)$ \\
\hline $13-36$ & $27(46,6)$ & $40(32,8)$ & $67(37,2)$ & $14(27,5)$ & $31(20,7)$ & $45(22,4)$ \\
\hline$>36$ & $10(17,2)$ & $64(52,5)$ & $74(41,4)$ & $25(49,0)$ & $97(64,7)$ & $122(60,7)$ \\
\hline \multicolumn{7}{|l|}{ Vínculo institucional } \\
\hline Programa Mais Médicos */PROVAB ** & $31(53,4)$ & $0(0,0)$ & $31(17,2)$ & $7(13,7)$ & $0(0,0)$ & $7(3,4)$ \\
\hline Emprego público estatutário & $13(22,4)$ & $94(77,0)$ & $107(59,4)$ & $36(70,6)$ & $103(68,7)$ & $139(69,2)$ \\
\hline Contrato por tempo determinado & $8(13,8)$ & $16(13,1)$ & $24(13,3)$ & $7(13,7)$ & $3(2,0)$ & $10(5,0)$ \\
\hline Contrato por tempo indeterminado & $5(8,6)$ & $11(9,0)$ & $16(5,6)$ & $1(2,0)$ & $19(12,7)$ & $20(10,0)$ \\
\hline
\end{tabular}

Nota: questões respondidas apenas pelos profissionais que utilizavam os mecanismos referidos e se encaixavam no perfil de resposta das perguntas. O quantitativo de respondentes variou de acordo com o mecanismo e com o nível de atenção que utiliza.

Fonte: elaboração própria.

* Programa Mais Médicos é um programa do Governo Federal que supera a barreira da ausência de médicos em localidades de alta vulnerabilidade e afastada da capital;

** PROVAB é o Programa de Valorização da Atenção Baśica, que é uma iniciativa do Governo Federal para interiorização e qualificação dos médicos na especialidade de medicina da família.

para o bilhete um maior percentual (51\%) na rede Caruaru, principalmente pelos médicos da atenção básica (72\%) (Tabela 3).

Em ambas as redes, o envio do formulário de referência e formulário de contrarreferência era realizado predominantemente (61,7\% rede Caruaru e 76,6\% rede Recife) pelo paciente, principalmente na atenção especializada. Vale ressaltar que metade dos profissionais na rede Recife referiu a central de regulação como forma de envio desses formulários. As informações preenchidas eram diferentes entre os níveis de atenção: na atenção básica, era o tratamento e diagnóstico, já na atenção especializada era motivo de encaminhamento e história clínica (Tabela 3). 


\section{Tabela 2}

Conhecimento (existência e finalidade de uso) sobre mecanismos de articulação entre níveis de atenção. Caruaru e Recife (DS III e VII), Pernambuco, Brasil, 2015

\begin{tabular}{|c|c|c|c|c|c|c|}
\hline & \multicolumn{3}{|c|}{ Caruaru } & \multicolumn{3}{|c|}{ Recife } \\
\hline & $\begin{array}{c}\text { Atenção } \\
\text { básica } \\
(n=58) \\
n(\%)\end{array}$ & $\begin{array}{c}\text { Atenção } \\
\text { especializada } \\
(n=122) \\
n(\%)\end{array}$ & $\begin{array}{c}\text { Total } \\
(\mathrm{N}=180) \\
\text { n (\%) }\end{array}$ & $\begin{array}{c}\text { Atenção } \\
\text { básica } \\
\text { (n= 51) } \\
\text { n (\%) }\end{array}$ & $\begin{array}{c}\text { Atenção } \\
\text { especializada } \\
(n=150) \\
n(\%)\end{array}$ & $\begin{array}{c}\text { Total } \\
(\mathrm{N}=201) \\
\mathrm{n}(\%)\end{array}$ \\
\hline \multicolumn{7}{|l|}{ Existência do mecanismo } \\
\hline \multicolumn{7}{|l|}{ Formulários de referência e de contrarreferência } \\
\hline Sim & $37(63,8)$ & $50(41,0)$ & $87(48,3)$ & $48(94,1)$ & $99(66,0)$ & $147(73,1)$ \\
\hline \multicolumn{7}{|l|}{ Resumo de alta hospitalar } \\
\hline Sim & $20(34,5)$ & $68(55,7)$ & $88(48,9)$ & $34(66,7)$ & $87(58,0)$ & $121(60,2)$ \\
\hline \multicolumn{7}{|l|}{$\begin{array}{l}\text { Protocolos do Ministério da Saúde } \\
\text { compartilhados entre níveis assistenciais }\end{array}$} \\
\hline Sim & $36(62,1)$ & $21(17,2)$ & $57(31,7)$ & $27(52,9)$ & $42(28,0)$ & $69(34,3)$ \\
\hline \multicolumn{7}{|l|}{ Sessões clínicas conjuntas } \\
\hline Sim & $9(15,6)$ & $3(2,5)$ & $12(6,7)$ & $12(23,5)$ & $9(6,0)$ & $21(10,4)$ \\
\hline \multicolumn{7}{|l|}{ Telefone institucional } \\
\hline Sim & $41(70,7)$ & $66(54,1)$ & $107(59,4)$ & $36(70,6)$ & $78(52,0)$ & $114(56,7)$ \\
\hline \multicolumn{7}{|l|}{ Finalidade de uso dos mecanismos * } \\
\hline Formulários de referência e de contrarreferência & $n=37$ & $n=43$ & $\mathrm{n}=80$ & $\mathrm{n}=48$ & $\mathrm{n}=93$ & $n=141$ \\
\hline Trocar informações & $24(64,8)$ & $36(83,7)$ & $60(75,0)$ & $37(77,0)$ & $62(66,7)$ & $99(70,2)$ \\
\hline Encaminhar o paciente & $11(29,7)$ & $6(13,9)$ & $17(21,5)$ & $15(31,3)$ & $34(36,6)$ & $49(34,8)$ \\
\hline Burocracia & $5(13,5)$ & $2(4,7)$ & $7(8,8)$ & $1(2,1)$ & $2(2,2)$ & $3(2,1)$ \\
\hline Resumo de alta hospitalar & $n=20$ & $n=68$ & $\mathrm{n}=88$ & $n=34$ & $n=87$ & $n=121$ \\
\hline Trocar informações & $16(80,0)$ & $61(89,7)$ & $77(87,5)$ & $33(97,0)$ & $70(80,4)$ & $103(85,1)$ \\
\hline Acompanhar o paciente & $10(50,0)$ & $18(26,4)$ & $28(31,8)$ & $14(41,2)$ & $28(32,2)$ & $41(33,9)$ \\
\hline $\begin{array}{l}\text { Protocolos do Ministério da Saúde } \\
\text { compartilhados entre níveis assistenciais }\end{array}$ & $n=36$ & $n=21$ & $n=57$ & $n=27$ & $n=42$ & $n=69$ \\
\hline Padronizar a atenção & $14(38,8)$ & $7(33,3)$ & $21(36,8)$ & $12(44,4)$ & $26(61,9)$ & $38(51,1)$ \\
\hline Qualidade da atenção & $10(27,7)$ & $3(14,2)$ & $13(22,8)$ & $6(22,2)$ & $5(11,9)$ & $11(15,9)$ \\
\hline Orientar o atendimento & $9(25,0)$ & $7(33,3)$ & $16(28,0)$ & $10(37,0)$ & $12(28,6)$ & $22(31,9)$ \\
\hline Sessões clínicas conjuntas & $\mathrm{n}=9$ & $\mathrm{n}=3$ & $\mathrm{n}=12$ & $\mathrm{n}=12$ & $\mathrm{n}=9$ & $\mathrm{n}=21$ \\
\hline Aprimorar o conhecimento & $5(55,5)$ & $2(66,6)$ & $7(58,3)$ & $7(58,3)$ & $4(44,4)$ & $11(52,4)$ \\
\hline Unificar a conduta & $2(2,2)$ & $1(33,3)$ & $3(25,0)$ & $1(8,3)$ & $0(0,0)$ & $1(4,8)$ \\
\hline Discussão de casos/Troca de experiências & $2(22,2)$ & $2(66,6)$ & $4(33,3)$ & $6(50,0)$ & $9(100,0)$ & $15(71,4)$ \\
\hline Bilhetes & $n=25$ & $n=71$ & $n=96$ & $n=27$ & $n=93$ & $n=120$ \\
\hline Trocar informações & $11(44,0)$ & $36(50,7)$ & $47(49,0)$ & $11(40,7)$ & $42(45,1)$ & $53(44,1)$ \\
\hline Encaminhar o paciente & $4(16,0)$ & $10(14,0)$ & $14(14,5)$ & $1(3,7)$ & $7(7,5)$ & $8(6,7)$ \\
\hline Ausência de mecanismos formais & $4(16,0)$ & $6(8,4)$ & $10(10,4)$ & $1(3,7)$ & $9(9,6)$ & $10(8,3)$ \\
\hline
\end{tabular}

Fonte: elaboração própria.

Nota: questões respondidas apenas pelos profissionais que utilizavam os mecanismos referidos e se encaixavam no perfil de resposta das perguntas. O quantitativo de respondentes variou de acordo com o mecanismo e com o nível de atenção que utiliza.

* Respostas múltiplas. 


\section{Figura 1}

Envio e recebimento do formulário de referência e de contrarreferência e do resumo de alta hospitalar. Caruaru e Recife (DS III e VII), Pernambuco, Brasil, 2015.

1a) Formulário de referência e de contrarreferência

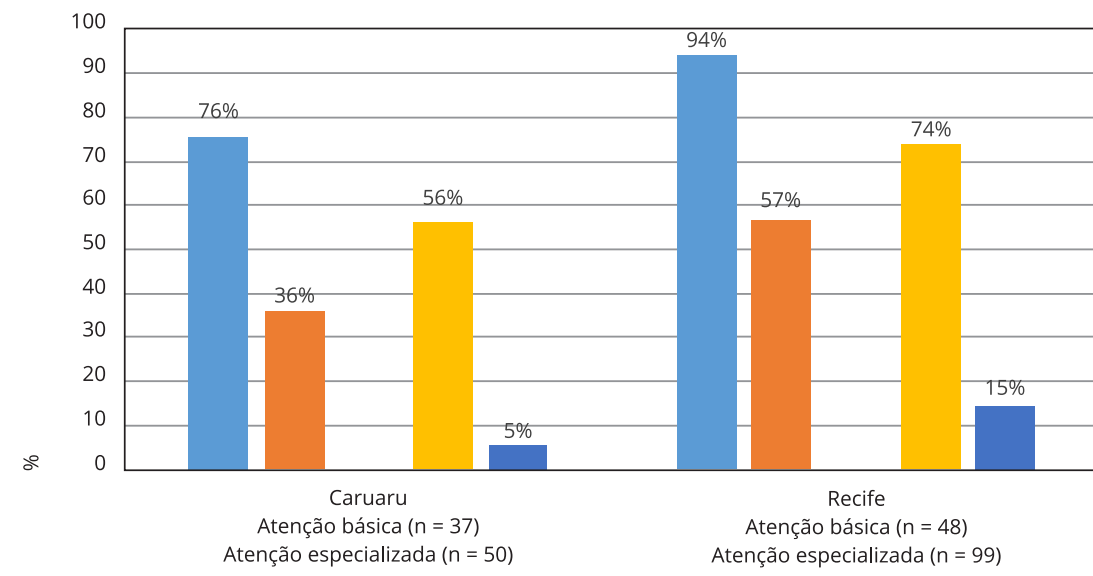

Atenção básica que envia o formulário de referência

Atenção especializada que recebe o formulário de referência

Atenção especilaizada que envia

o formulário de contrarreferência

Atenção básica que recebe o formulário de contrarreferência

1b) Resumo de alta hospitalar

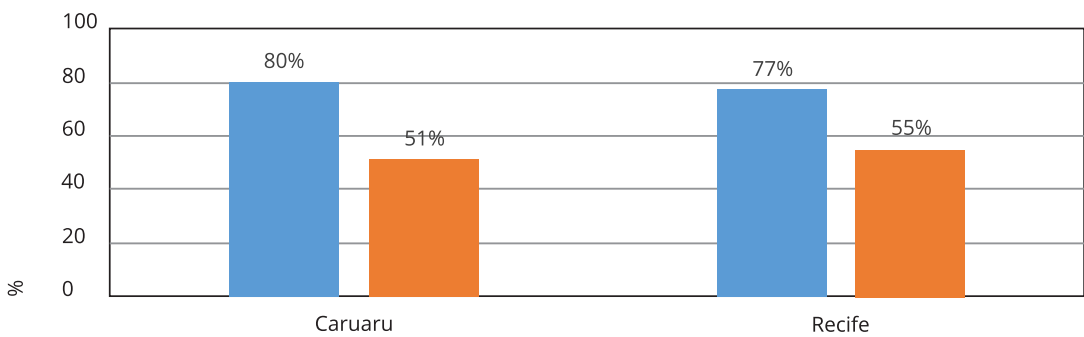

Atenção especializada que envia o resumo de alta hospitalar

Atenção básica que recebe o resumo de alta hospitalar

Quanto ao resumo de alta hospitalar, em ambas as redes, as principais informações contidas foram: tratamento farmacológico e diagnóstico nos dois níveis de atenção. Nas duas redes, os protocolos do Ministério da Saúde utilizados eram diferentes e de acordo com o nível de atenção. Para os médicos da atenção básica os mais utilizados eram pré-natal e tuberculose/hanseníase, já na atenção especializada eram doenças infectocontagiosas e dengue. Os médicos da atenção básica afirmaram participar mais das capacitações para uso dos protocolos do que os da atenção especilaizada, nas duas redes estudadas (Tabela 3 ).

Segundo os médicos da atenção básica de Caruaru, as especialidades que mais participavam das sessões clínicas conjuntas eram: ginecologia e obstetrícia (55,5\%) e psiquiatria (33,3\%). Já na atenção especializada eram os médicos da família (99\%). Em Recife, a especialidade mais referida foi dermatologia na atenção básica (50\%) e psiquiatria (55,6\%), e médicos da família $(55,6 \%)$ na atenção especializada. No que se refere ao tempo disponível para a realização da articulação clínica (preencher formulários, responder bilhetes, revisar protocolos), os médicos da atenção básica de ambas as redes referiram ter tempo disponível para isto, ao contrário dos especialistas que afirmaram não ter tempo (Caruaru 41,3\% e Recife 47\%). 
Tabela 3

Frequência e características de utilização dos mecanismos de articulação entre níveis de atenção. Caruaru e Recife (DS III e VII), Pernambuco, Brasil, 2015

\begin{tabular}{|c|c|c|c|c|c|c|}
\hline & $\begin{array}{l}\text { Atenção } \\
\text { básica } \\
\text { n (\%) }\end{array}$ & $\begin{array}{c}\text { Caruaru } \\
\text { Atenção } \\
\text { especializada } \\
n(\%)\end{array}$ & $\begin{array}{l}\text { Total } \\
\text { n (\%) }\end{array}$ & $\begin{array}{l}\text { Atenção } \\
\text { básica } \\
\text { n (\%) }\end{array}$ & $\begin{array}{c}\text { Recife } \\
\text { Atenção } \\
\text { especializada } \\
\mathbf{n}(\%)\end{array}$ & $\begin{array}{l}\text { Total } \\
\text { n (\%) }\end{array}$ \\
\hline \multicolumn{7}{|l|}{ Frequência de uso } \\
\hline $\begin{array}{l}\text { Seguimento dos protocolos do Ministério } \\
\text { da Saúde compartilhados entre níveis } \\
\text { assistenciais }\end{array}$ & $\mathrm{n}=36$ & $\mathrm{n}=20$ & $n=56$ & $n=27$ & $\mathrm{n}=41$ & $n=68$ \\
\hline Alto & $35(97,2)$ & $17(85,0)$ & $52(92,9)$ & $26(96,2)$ & $38(92,7)$ & $64(94,1)$ \\
\hline Participação nas sessões clínicas conjuntas & $\mathrm{n}=9$ & $n=3$ & $\mathrm{n}=12$ & $\mathrm{n}=12$ & $\mathrm{n}=9$ & $\mathrm{n}=21$ \\
\hline Alto & $8(88,9)$ & $0(0,0)$ & $8(66,7)$ & $8(66,7)$ & $6(66,7)$ & $14(66,7)$ \\
\hline Telefone institucional & $\mathrm{n}=22$ & $n=66$ & $\mathrm{n}=88$ & $\mathrm{n}=30$ & $\mathrm{n}=79$ & $n=109$ \\
\hline Alto & $9(41,0)$ & $27(41,0)$ & $36(41,0)$ & $10(33,3)$ & $34(43,0)$ & $44(40,3)$ \\
\hline Bilhetes & $\mathrm{n}=25$ & $\mathrm{n}=71$ & $\mathrm{n}=96$ & $\mathrm{n}=27$ & $\mathrm{n}=93$ & $n=120$ \\
\hline Alto & $18(72,0)$ & $31(43,7)$ & $49(51,0)$ & $6(22,2)$ & $36(38,8)$ & $42(35,0)$ \\
\hline \multicolumn{7}{|l|}{ Características de utilização } \\
\hline $\begin{array}{l}\text { Forma de envio do dos formulários de } \\
\text { referência e contrarreferência }\end{array}$ & $\mathrm{n}=37$ & $n=44$ & $\mathrm{n}=81$ & $\mathrm{n}=48$ & $n=93$ & $\mathrm{n}=141$ \\
\hline Pelo paciente & $19(51,4)$ & $31(70,5)$ & $50(61,7)$ & $23(47,9)$ & $85(91,4)$ & $108(76,6)$ \\
\hline Pela regulação & $12(32,4)$ & $9(20,5)$ & $21(25,9)$ & $24(50,0)$ & $6(6,5)$ & $30(21,3)$ \\
\hline Outros & $8(21,6)$ & $6(13,6)$ & $14(17,2)$ & $2(4,1)$ & $3(3,2)$ & $5(3,5)$ \\
\hline $\begin{array}{l}\text { Informações contidas no formulário de } \\
\text { referência }\end{array}$ & $n=18$ & & & $n=34$ & & \\
\hline Tratamentos & $16(88,8)$ & & & $26(76,5)$ & & \\
\hline Diagnóstico & $14(77,7)$ & & & $24(70,6)$ & & \\
\hline Dados pessoais & $9(50,0)$ & & & $2(5,9)$ & & \\
\hline História clínica & $8(44,4)$ & & & $7(20,6)$ & & \\
\hline Exames & $7(38,8)$ & & & $7(20,6)$ & & \\
\hline Motivo do encaminhamento & $2(11,1)$ & & & $4(11,8)$ & & \\
\hline $\begin{array}{l}\text { Informações contidas no formulário de } \\
\text { contrarreferência }\end{array}$ & & $n=36$ & & & $\mathrm{n}=90$ & \\
\hline Tratamentos & & $16(44,4)$ & & & $17(18,9)$ & \\
\hline Diagnóstico & & $19(52,7)$ & & & $38(42,2)$ & \\
\hline Dados pessoais & & $14(38,8)$ & & & $17(18,9)$ & \\
\hline História clínica & & $19(52,7)$ & & & $44(48,9)$ & \\
\hline Exames & & $9(25,0)$ & & & $15(16,7)$ & \\
\hline Motivo do encaminhamento & & $13(36,1)$ & & & $48(53,3)$ & \\
\hline $\begin{array}{l}\text { Recebimento de informações em tempo } \\
\text { útil (formulário de contrarreferência) }\end{array}$ & $\mathrm{n}=18$ & & & $n=34$ & & \\
\hline $\operatorname{Sim}$ & $7(38,9)$ & & & $9(26,4)$ & & \\
\hline Não & $11(61,1)$ & & & $25(73,5)$ & & \\
\hline $\begin{array}{l}\text { Informações contidas no resumo de alta } \\
\text { hospitalar }\end{array}$ & $n=18$ & & & $n=33$ & & \\
\hline Tratamento farmacológico & $14(77,8)$ & & & $24(72,7)$ & & \\
\hline Diagnóstico & $13(72,2)$ & & & $26(78,8)$ & & \\
\hline Motivo de internação & $12(66,7)$ & & & $15(45,5)$ & & \\
\hline Procedimentos realizados & $11(61,1)$ & & & $20(60,6)$ & & \\
\hline Resultados de exames & $6(33,3)$ & & & $17(51,5)$ & & \\
\hline Acompanhamento & $6(33,3)$ & & & $14(42,4)$ & & \\
\hline
\end{tabular}

(continua) 


\begin{tabular}{|c|c|c|c|c|c|c|}
\hline & & Caruaru & & & Recife & \\
\hline & $\begin{array}{c}\text { Atenção } \\
\text { básica } \\
\text { n (\%) }\end{array}$ & $\begin{array}{c}\text { Atenção } \\
\text { especializada } \\
n(\%)\end{array}$ & $\begin{array}{l}\text { Total } \\
\text { n (\%) }\end{array}$ & $\begin{array}{l}\text { Atenção } \\
\text { básica } \\
\text { n (\%) }\end{array}$ & $\begin{array}{c}\text { Atenção } \\
\text { especializada } \\
\mathbf{n}(\%)\end{array}$ & $\begin{array}{l}\text { Total } \\
\text { n (\%) }\end{array}$ \\
\hline \multicolumn{7}{|l|}{ Características de utilização } \\
\hline Protocolos utilizados & $\mathrm{n}=36$ & $\mathrm{n}=21$ & $\mathrm{n}=57$ & $\mathrm{n}=27$ & $n=42$ & $\mathrm{n}=69$ \\
\hline Tuberculose/Hanseníase & $12(33,3)$ & $5(23,8)$ & $17(29,8)$ & $12(44,4)$ & $7(16,7)$ & $19(27,5)$ \\
\hline Pré-natal & $16(44,4)$ & $0(0,0)$ & $16(28,0)$ & $10(37,0)$ & $2(4,7)$ & $12(17,3)$ \\
\hline Dengue & $8(22,2)$ & $7(33,3)$ & $15(26,3)$ & $5(18,5)$ & $17(40,4)$ & $22(31,8)$ \\
\hline Doenças infectocontagiosas & $7(19,4)$ & $8(38,0)$ & $14(24,5)$ & $10(37,0)$ & $16(38,0)$ & $26(37,7)$ \\
\hline Criança & $11(30,5)$ & $3(14,2)$ & $14(24,5)$ & $10(37,0)$ & $9(21,4)$ & $19(27,5)$ \\
\hline DCNT & $11(30,5)$ & $0(0,0)$ & $11(19,2)$ & $15(55,5)$ & $2(4,7)$ & $17(24,6)$ \\
\hline $\begin{array}{l}\text { Participação em capacitações para } \\
\text { utilização dos protocolos }\end{array}$ & $n=36$ & $n=21$ & $n=57$ & $n=27$ & $\mathrm{n}=42$ & $\mathrm{n}=69$ \\
\hline Sim & $23(63,9)$ & $10(47,6)$ & $33(57,9)$ & $16(59,3)$ & $23(54,8)$ & $39(56,5)$ \\
\hline
\end{tabular}

DCNT: doença crônica não transmissível.

Fonte: elaboração própria.

Nota: questões respondidas apenas pelos profissionais que utilizavam os mecanismos referidos e se encaixavam no perfil de resposta das perguntas. O quantitativo de respondentes variou de acordo com o mecanismo e com o nível de atenção que utiliza.

Para o formulário de referência, formulário de contrarreferência e resumo de alta, a maioria dos respondentes afirmou não ter dificuldades no uso destes mecanismos em ambos os níveis e nas duas redes de estudo. A exceção foi na utilização das sessões clínicas conjuntas, para as quais os especialistas de ambas as redes referiram dificuldades, especialmente na rede Caruaru. Os médicos da atenção básica explicitaram menos dificuldades na utilização dos mecanismos quando comparados com os da atenção especializada. Em relação às dificuldades referidas no sistema de referência/contrarreferência e resumo de alta, o preenchimento inadequado/informações incompletas e o baixo recebimento destes documentos foram citados em ambas as redes (principalmente na rede Caruaru). Em relação às sessões clínicas conjuntas, para as quais os especialistas de ambas as redes referiram dificuldades de uso, especialmente na rede Caruaru, as questões apontadas foram: dificuldade em combinar horários e a pouca participação dos profissionais médicos. Para os protocolos foi a deficiência na estrutura dos serviços e a dificuldade na realização dos exames (Tabela 4).

\section{Discussão}

Os resultados encontrados evidenciaram falhas na articulação entre níveis nas duas redes avaliadas e problemas na implantação dos mecanismos, achados recorrentes na literatura 13,14,15,16. Porém, os entraves não ocorreram da mesma forma nas duas redes e é notória a maior participação da atenção básica do que a atenção especializada em promover a coordenação/articulação do cuidado. Na comparação entre redes, observa-se que na rede Recife os médicos conhecem e utilizam mais mecanismos do que na rede Caruaru. Recife é a grande metrópole do estado e da região, e assim como outros centros urbanos conta com melhores arcabouços estruturais e processuais que podem influenciar positivamente na coordenação/articulação da atenção à saúde.

A implementação dos mecanismos para articulação entre níveis ainda apresenta deficiências em ambas as redes. Essas apontam para possíveis entraves no desenvolvimento das políticas públicas brasileiras e na presença de falhas na organização das redes em saúde 15,16,17,18. 
Tabela 4

Dificuldades na utilização dos mecanismos de articulação entre níveis de atenção. Caruaru e Recife (DS III e VII), Pernambuco, Brasil, 2015.

\begin{tabular}{|c|c|c|c|c|c|c|}
\hline & $\begin{array}{l}\text { Atenção } \\
\text { básica } \\
\text { n (\%) }\end{array}$ & $\begin{array}{c}\text { Caruaru } \\
\text { Atenção } \\
\text { especializada } \\
\text { n (\%) }\end{array}$ & $\begin{array}{l}\text { Total } \\
\text { n (\%) }\end{array}$ & $\begin{array}{l}\text { Atenção } \\
\text { básica } \\
\text { n (\%) }\end{array}$ & $\begin{array}{c}\text { Recife } \\
\text { Atenção } \\
\text { especializada } \\
\text { n (\%) }\end{array}$ & $\begin{array}{l}\text { Total } \\
\text { n (\%) }\end{array}$ \\
\hline \multicolumn{7}{|l|}{ Existência de dificuldades } \\
\hline $\begin{array}{l}\text { Formulários de referência e } \\
\text { contrarreferência }\end{array}$ & $\mathrm{n}=37$ & $\mathrm{n}=50$ & $\mathrm{n}=87$ & $n=48$ & $\mathrm{n}=99$ & $\mathrm{n}=147$ \\
\hline Sim & $6(16,2)$ & $14(28,0)$ & $20(23,0)$ & $12(25,0)$ & $24(24,2)$ & $36(24,5)$ \\
\hline Resumo de alta hospitalar & $n=20$ & $n=69$ & $\mathrm{n}=89$ & $n=34$ & $\mathrm{n}=87$ & $\mathrm{n}=131$ \\
\hline Sim & $7(35,0)$ & $17(24,6)$ & $24(26,9)$ & $3(8,8)$ & $14(16,1)$ & $17(14,0)$ \\
\hline $\begin{array}{l}\text { Protocolos do Ministério da Saúde } \\
\text { compartilhados entre níveis } \\
\text { assistenciais }\end{array}$ & $n=36$ & $n=21$ & $\mathrm{n}=57$ & $n=27$ & $n=42$ & $\mathrm{n}=69$ \\
\hline Sim & $6(16,7)$ & $4(19,0)$ & $10(17,5)$ & $5(18,5)$ & $12(28,6)$ & $17(24,6)$ \\
\hline Sessões clínicas conjuntas & $\mathrm{n}=9$ & $\mathrm{n}=3$ & $\mathrm{n}=12$ & $\mathrm{n}=12$ & $\mathrm{n}=9$ & $n=21$ \\
\hline Sim & $1(11,1)$ & $3(100,0)$ & $4(33,3)$ & $3(25,0)$ & $8(88,8)$ & $11(52,54)$ \\
\hline \multicolumn{7}{|l|}{ Dificuldades referidas } \\
\hline $\begin{array}{l}\text { Formulários de referência e } \\
\text { contrarreferência }\end{array}$ & $\mathrm{n}=6$ & $n=14$ & $\mathrm{n}=20$ & $\mathrm{n}=12$ & $n=24$ & $n=36$ \\
\hline Informações incompletas & $3(50,0)$ & $5(35,7)$ & $8(40,0)$ & $2(16,7)$ & $12(50,0)$ & $14(38,9)$ \\
\hline $\begin{array}{l}\text { Não recebem o formulário de } \\
\text { contrarreferência }\end{array}$ & $2(33,3)$ & $2(14,3)$ & $4(20,0)$ & $3(25,0)$ & $3(12,5)$ & $6(16,7)$ \\
\hline Burocracia/Formulário longo & $2(33,3)$ & $6(42,8)$ & $8(40,0)$ & $5(41,6)$ & $4(16,6)$ & $9(25,0)$ \\
\hline Resumo de alta hospitalar & $n=7$ & $\mathrm{n}=17$ & $n=24$ & $n=3$ & $n=14$ & $\mathrm{n}=17$ \\
\hline Informações incompletas & $5(71,4)$ & $13(76,4)$ & $18(78,0)$ & $2(66,7)$ & $8(57,1)$ & $10(58,8)$ \\
\hline Letra ilegível & $2(28,5)$ & $4(23,5)$ & $6(25,0)$ & $1(33,3)$ & $2(14,3)$ & $3(17,6)$ \\
\hline Falta de informatização & $1(14,2)$ & $1(5,8)$ & $2(8,3)$ & $1(33,3)$ & $0(0,0)$ & $1(5,9)$ \\
\hline $\begin{array}{l}\text { Ausência do resumo de alta } \\
\text { hospitalar }\end{array}$ & $1(14,2)$ & $1(5,8)$ & $2(8,3)$ & $0(0,0)$ & $2(14,3)$ & $2(11,8)$ \\
\hline $\begin{array}{l}\text { Protocolos do Ministério da Saúde } \\
\text { compartilhados entre níveis } \\
\text { assistenciais }\end{array}$ & $\mathrm{n}=6$ & $\mathrm{n}=4$ & $n=10$ & $n=5$ & $n=12$ & $\mathrm{n}=17$ \\
\hline Deficiência na estrutura dos serviços & $2(33,3)$ & $1(25,0)$ & $3(30,0)$ & $3(60,0)$ & $4(33,3)$ & $7(41,2)$ \\
\hline Desconhecimento dos protocolos & $3(50,0)$ & $2(50,0)$ & $5(50,0)$ & $0(0,0)$ & $2(16,7)$ & $2(11,8)$ \\
\hline Dificuldade na realização de exames & $3(50,0)$ & $0(0,0)$ & $3(30,0)$ & $1(20,0)$ & $4(33,3)$ & $5(29,4)$ \\
\hline Sessões clínicas conjuntas & $\mathrm{n}=1$ & $\mathrm{n}=3$ & $n=4$ & $\mathrm{n}=3$ & $\mathrm{n}=8$ & $\mathrm{n}=11$ \\
\hline Dificuldades em aceitar um horário & $1(100,0)$ & $1(33,3)$ & $2(50,0)$ & $0(0,0)$ & $6(75,0)$ & $6(54,5)$ \\
\hline Ausência dos médicos & $0(0,0)$ & $1(33,3)$ & $1(25,0)$ & $0(0,0)$ & $4(50,0)$ & $4(36,3)$ \\
\hline
\end{tabular}

Fonte: elaboração própria.

Nota: questões respondidas apenas pelos profissionais que utilizavam o mecanismo referido e apontavam dificuldades no uso. $\mathrm{O}$ quantitativo de respondentes variou de acordo com o mecanismo.

\section{Características sociodemográficas}

No que se refere às características sociodemográficas, os grupos de respondentes apresentaram-se semelhantes, exceto quanto à faixa etária, com $50 \%$ dos profissionais da atenção básica na rede Recife com 50 anos ou mais. Apesar de não haver consenso, a idade pode influenciar no conhecimento e uso de mecanismos para a articulação entre os níveis, haja vista que profissionais com mais experiência e com o amadurecimento pessoal podem ser mais críticos e menos tolerantes a mudanças 11,13,15. 
Os vínculos empregatícios mais prevalentes na atenção básica da rede Caruaru foram Programa Mais Médicos e PROVAB, fato que pode estar relacionado com a presença de médicos cubanos que representam um papel relevante na amostra desta rede. O Programa Mais Médicos foi institucionalizado em 2013, como uma iniciativa do Governo Federal para superar a escassez e a rotatividade dos médicos (principalmente em municípios pequenos e em áreas mais vulneráveis) e investir no direcionamento da formação voltada para prática na atenção básica 19. Esses profissionais têm uma visão mais crítica e mais holística da saúde em comunidades, podendo em alguns aspectos se diferenciar da formação médica brasileira tradicional inicial, o que pode influenciar no conhecimento e no uso dos mecanismos.

Quanto ao tempo de experiência na rede, os médicos de Caruaru tinham um menor tempo de trabalho referido quando comparados a outra rede estudada, em ambos os níveis. Esses achados podem sugerir que redes afastadas dos grandes centros urbanos teriam uma maior rotatividade de profissionais, fato que pode contribuir para a desvalorização e um menor interesse na qualidade da atenção prestada, que está intimamente ligada ao uso dos mecanismos 19,20. Essa diferença entre capital e interior também pode justificar a melhor qualificação profissional dos médicos da atenção básica recifenses (quase $40 \%$ dos médicos da atenção básica de Recife possuem pós-graduação em medicina de família e comunidade, comparados aos $20 \%$ dos médicos de Caruaru). Estudos 21,22 mostram que áreas mais afastadas e com baixa renda apresentam dificuldades na contratação de profissionais mais qualificados, que, quando contratados, mantêm vínculos frágeis que permitem a mudança de emprego com mais facilidade. Esse perfil de profissionais especializados em suas áreas de atuação, principalmente na atenção básica, favorece a melhoria da coordenação da atenção e a qualidade dos serviços prestados, que pode estar relacionada à utilização mais eficiente dos mecanismos de articulação entre níveis.

\section{Mecanismos de adaptação mútua}

Dentre os mecanismos de adaptação mútua, os formulários de referência e contrarreferência são considerados os principais mecanismos e os mais comuns para a integração dos diferentes níveis de atenção à saúde, e muitas vezes são os únicos existentes, principalmente em algumas regiões do Brasil $20,21,23$. No presente estudo, os médicos referiram o repasse de informações para o outro nível como sua principal finalidade, concordando com os estudos de Vermeir et al. 18, Durbin et al. 24, Reig-Mollá et al. ${ }^{22}$. Vale destacar o papel administrativo desse formulário, finalidade também referida neste estudo e que pode estar relacionada à baixa qualidade de preenchimento, desvalorização de utilização e o uso apenas burocrático desse mecanismo 14,18 .

Entre as redes, os profissionais da rede Recife conhecem e utilizam mais o formulário de referência e o formulário de contrarreferência do que os de Caruaru. Todavia, esses formulários são subutilizados, visto que quase metade dos médicos da rede Caruaru e um quarto da amostra da rede Recife não faz uso desses mecanismos, concordando com os estudos de Stille et al. 15, Vermeir et al. 18, O’Malley \& Reschovsky 23 e Berendsen et al. 20. Quando comparados entre níveis, são os médicos da atenção especializada que menos utilizam, especialmente na rede Recife onde a discrepância é ainda maior, sugerindo divergências e impasses no uso do formulário de contrarreferência 14,20,22,25,26,27. Essa baixa utilização se torna mais evidente ao se analisar a frequência de envio e recebimento desses documentos, com uma alta discrepância nas duas redes de atenção, principalmente quanto ao formulário de contrarreferência.

Em outros estudos 17,21,22,25 também foram encontradas divergências, que revelam baixos percentuais de recebimento do formulário de contrarreferência. Esse cenário alerta para a utilização apenas burocrática desses formulários, contrapondo com a sua real finalidade de repassar informações úteis que contribuam com a qualidade da atenção e comunicação entre os profissionais $20,25,27$. Vale ressaltar que nas duas redes estudadas é obrigatória a utilização desse mecanismo para o encaminhamento do paciente ao especialista por meio de políticas aprovadas e consolidadas em nível nacional 27,28,29.

A incipiência da contrarreferência gera uma quebra na continuidade do cuidado, contribuindo para fragilizar o papel da atenção básica como coordenadora e ordenadora da rede de serviços 17,20,29. Apesar de subutilizado, a maioria dos entrevistados não identificou dificuldades no instrumento. $\mathrm{O}$ baixo aproveitamento desse mecanismo pode ser consequência da alta rotatividade dos profissionais, o que contribui negativamente para a qualidade da atenção 13,20,23. 
A principal informação que deve estar contida nesses formulários é o motivo da referência 8,14,27. Entretanto, essa resposta foi pouco citada pelos entrevistados. Ainda, informações sobre resultados de exames também foram pouco citadas em ambas as redes estudadas. São informações reconhecidamente relevantes para a tomada de decisão e para evitar perda de tempo e duplicações desnecessárias 8,20,27.

O resumo de alta hospitalar é o principal mecanismo de comunicação entre a atenção básica e o hospital, porém é pouco usado, como verfificado nos resultados do presente estudo e confirmado na literatura 28,29. Vale salientar que neste estudo foi considerado o envio do resumo de alta hospitalar por especialistas que atendiam pacientes internados nas unidades de urgência/emergência. Quanto ao envio e recebimento do mesmo, existe uma discrepância entre níveis, sugerindo que quando é enviado, geralmente não é recebido. Isso pode ocorrer devido à ausência de um repasse formal e institucionalizado que realize a troca de informações para os diferentes níveis. Muitas vezes, o usuário é o principal responsável pelo envio e recebimento de alguns mecanismos de um nível para outro. Essa prática pode gerar falsificações e alterações no documento, erros médicos, além da perda e desvio dos formulários 16,17 .

Por outro lado, existe a Central de Regulação, uma estrutura operacional que gerencia a relação entre a demanda, oferta e informações dos serviços de saúde existentes na rede e que tem como finalidade organizar os fluxos de pessoas, produtos e informações ao longo das redes 26 . A Central de Regulação foi citada como mecanismo em ambas as redes (2,4\% na rede Caruaru e 2\% na rede Recife), porém com percentuais muito baixos, podendo indicar a não identificação desta ferramenta como um mecanismo de articulação ou a baixa implementação deste serviço. Com o correto funcionamento e utilização dessa Central, que seria a principal responsável por esse intercâmbio das informações, os fluxos ficariam estabelecidos de forma institucional, reduzindo a influência de fatores externos.

Quanto à presença de mecanismos de comunicação informal, a sua utilização pode indicar a presença de lacunas na articulação entre níveis que não foram sanadas pelos métodos formais, sugerindo uma deficiência na implementação destes. O telefone e os bilhetes são amplamente usados nas duas redes de estudo e servem para os profissionais trocar informações clínicas sobre os pacientes. Além desses, o WhatsApp (2,6\%) também foi citado como mecanismo entre níveis. Em Vázquez et al. ${ }^{14}$, os bilhetes eram utilizados para realizar encaminhamentos entre níveis, devido à baixa utilização $\mathrm{e}$ implantação de mecanismos formais que realizariam esta função. Neste estudo, esses bilhetes têm uma função maior que o encaminhamento de pacientes entre níveis, servem como forma de comunicação entre os médicos, e seu uso é justificado pela praticidade, facilidade e pela inexistência de mecanismos formais. Esses achados podem indicar que o fluxo de informações não está a contento e também a presença de falhas na implantação de mecanismos formais 8,13,14.

\section{Mecanismos de padronização}

As políticas públicas no Brasil dão o arcabouço normativo para a criação e implementação de mecanismos que garantam a comunicação entre os diferentes níveis assistenciais, como as linhas de cuidado, os protocolos de atenção à saúde e as sessões clínicas conjuntas. A criação desses novos formatos de ferramentas é necessária e já ocorre em algumas redes de serviços, principalmente na Europa e na América do Norte 12.

No presente estudo, identificou-se que os protocolos do Ministério da Saúde são subutilizados pelos profissionais entrevistados das redes estudadas, achado este que está em concordância com o trabalho de Serra \& Rodrigues 21. Essa subutilização pode estar associada à pouca participação dos médicos em capacitações sobre os protocolos. Os resultados do estudo atual mostram que a atenção básica os utiliza, ainda que pouco, em maior quantidade e frequência do que a atenção especializada, e esta divergência entre os níveis de atenção sugere que estes mecanismos não estão sendo compartilhados 26,30 .

A principal função dos protocolos compartilhados, apontada pelos médicos de ambas as redes, é a padronização da atenção. Esse achado corrobora Serra \& Rodrigues 21 e outros estudos 14,16,30 que justificaram o uso destes protocolos para avaliar a necessidade de encaminhamentos, adequar as referências e favorecer a comunicação entre os prestadores de serviços. Em relação aos tipos de protocolos utilizados, eles foram diferentes entre os níveis e entre as redes estudadas, sugerindo prio- 
ridades distintas, devido às demandas e necessidades particulares. Essa diferença se contrapõe à ideia de compartilhamento entre níveis que deveria estar atrelada à utilização dessa ferramenta.

A sessão clínica conjunta foi o mecanismo menos conhecido e utilizado em ambos os níveis e em ambas as redes de atenção. Esse achado sugere uma implantação insuficiente e reforça a problemática envolvendo a pouca confiança do especialista no médico da atenção básica, que está relacionada à cultura de supremacia da atenção especializada e da participação dos médicos clínicos, durante as sessões conjuntas, como receptores de informações. A utilização desses espaços de forma simultânea, voluntária e adequada para as necessidades locais, construídos por meio de um processo participativo contínuo, contribui para a eficácia dos mesmos e a mudança de valores entre níveis 4,20.

Estudos 26,27 mostram que a existência de mecanismos não garante a sua utilização. Um dos investimentos para a melhoria da articulação entre níveis é a informatização dos serviços e das informações $20,22,26$. Porém, informatizar não garante o aumento do uso, visto que existem várias barreiras operacionais que não são sanadas com o uso da tecnologia. Essa inovação tecnológica despende altos custos de implantação e de manutenção, e nem sempre proporciona os resultados esperados 10,26.

Quando questionados sobre possíveis dificuldades na utilização dos mecanismos, a maioria dos respondentes referiu não ter problemas para usar essas ferramentas. Ainda assim, os mecanismos não estão sendo utilizados como deveriam. Em países desenvolvidos, onde o uso dos mesmos está implantado há algum tempo, também se identificaram impedimentos e entraves que diminuíram seu uso $8,27,31$.

Fatores como falta de habilidade na utilização, mecanismos mal implementados, experiências negativas anteriores, alta rotatividade de profissionais, desinteresse e descompromisso com a rede, recursos inadequados e falta de tempo para os registros são apontados como barreiras de utilização, e sua identificação pode auxiliar no planejamento de medidas futuras 14,27,30. A falta de interesse, de conscientização e de confiança nos médicos da atenção básica também são explicações possíveis para a subutilização dos mecanismos e estão relacionadas com condições precárias de trabalho, com a cultura de supervalorização dos especialistas e com o perfil dos profissionais da assistência 8,13,16.

A falta de tempo dos profissionais é uma das principais explicações para a baixa utilização ou a não utilização de alguns mecanismos 8,13,14. O estudo em tela concorda com os achados da literatura $16,17,27$, principalmente quanto aos especialistas que afirmaram não ter tempo útil, durante o período da consulta, para a realização da articulação clínica. Essa situação pode estar ligada à alta demanda de pacientes, à remuneração por produtividade e prioridades diferentes entre os médicos da atenção básica e atenção especializada 13,17 .

\section{Limitações do estudo}

Houve dificuldades para a discussão dos achados em decorrência da escassez de estudos prévios que abordassem a percepção dos profissionais sobre os mecanismos e sobre a existência e utilização das diversas ferramentas com foco na articulação entre os níveis.

\section{Conclusão}

Os resultados mostraram que, apesar de serem duas redes distintas (uma capital do estado e outra município do interior), os problemas explicitam falhas na implementação dos mecanismos, provocando lacunas na organização das redes de saúde. Os mecanismos estudados são subutilizados, muitas vezes inexistentes e quando usados apresentam divergências entre níveis, sugerindo a compartimentalização da ferramenta e não um compartilhamento da mesma. Apesar desse cenário, os mecanismos são mais conhecidos e utilizados na atenção básica do que na atenção especializada e na rede de Recife do que na de Caruaru.

O baixo conhecimento, a subutilização, a divergência de informações, discrepâncias no envio e recebimento entre níveis e o uso inadequado dos mecanismos sugerem falhas na comunicação entre os profissionais e entre níveis. Tal fato contribui para a fragmentação do cuidado, para deficiências na qualidade da atenção e dificulta a implantação adequada das políticas públicas. Investimentos em 
educação continuada que considerem os diferentes perfis e necessidades profissionais, com a realização de metodologias ativas, participativas e que estimulem o protagonismo entre esses atores, são estratégias que podem melhorar o funcionamento das RAS.

Apesar de não ser garantia na melhora da articulação entre níveis, o uso de prontuários únicos e informatizados em toda a rede de atenção representa uma contribuição importante. Para melhorar a utilização dos mecanismos na rede é imprescindível organizar o processo de trabalho que contemple um tempo destinado à articulação clínica e instituir espaços de encontro entre profissionais que propiciem uma melhora na comunicação, na confiança e no conhecimento entre eles. Os mecanismos são ferramentas prioritárias na busca da colaboração entre profissionais, que podem gerar melhorias para a integralidade do cuidado e na qualidade da atenção.

\section{Colaboradores}

C. R. F. Oliveira participou da concepção, coleta e análise dos dados, redação do artigo, revisão e aprovação da versão final. I. C. Samico e M. F. M. Mendes colaboraram na concepção, análise dos dados, redação do artigo, revisão e aprovação da versão final. I. Vargas e M. L. Vázquez contribuíram na análise dos dados, revisão e aprovação da versão final do artigo.

\section{Informações adicionais}

ORCID: Cecylia Roberta Ferreira de Oliveira (0000-0001-6960-5815); Isabella Chagas Samico (0000-0002-8338-7946); Marina Ferreira de Medeiros Mendes (0000-0002-5752-5217); Ingrid Vargas (0000-0002-1778-2411); Maria Luisa Vázquez (0000-0002-6091-8193).

\section{Agradecimentos}

À Comissão Europeia (Seventh Framework Programme - FP7-Health-2012-Innovation-1, number 305197) pelo financiamento.

\section{Referências}

1. Almeida PF, Giovanella L, Mendonça MHM, Escorel S. Desafios à coordenação dos cuidados em saúde: estratégias de integração entre níveis assistenciais em grandes centros urbanos. Cad Saúde Pública 2010; 26:286-98.

2. Mendes EV. As redes de atenção à saúde. Brasília: Organização Pan-Americana da Saúde; 2011.

3. Pan American Health Organization. Renewing primary health care in the Americas: concepts, policy options and a road map for implementation in the Americas. Washington DC: Pan American Health Organization; 2010.

4. Protasio APL, Silva PBD, Lima ECD, Gomes LB, Machado LDS, Valença AMG. Avaliação do sistema de referência e contrarreferência do Estado da Paraíba segundo os profissionais da atenção básica no contexto do 1o ciclo de avaliação externa do PMAQ-AB. Saúde Debate 2014; 38:209-20.

5. World Health Organization. Global status report on noncommunicable diseases. Geneva: World Health Organization; 2014.

6. Brasil. Decreto no 7.508 , de 28 de junho de 2011. Regulamenta a Lei no 8.080, de 19 de setembro de 1990, para dispor sobre a organização do Sistema Único de Saúde - SUS, o planejamento da saúde, a assistência à saúde e a articulação interfederativa e dá outras providências. Diário Oficial da União 2011; 28 jun.

7. Aguilera SLVU, França BHS, Moysés ST, Moysés SJ. Articulação entre os níveis de atenção dos serviços de saúde na Região Metropolitana de Curitiba: desafios para os gestores. Rev Adm Pública 2013; 47:1021-40. 
8. Terraza Núñez R, Vargas Lorenzo I, Vázquez Navarrete ML. La coordinación entre niveles asistenciales: una sistematización de sus instrumentos y medidas. Gac Sanit 2006; 20:48595.

9. Hartz ZMA, Contandriopoulos A-P. Integralidade da atenção e integração de serviços de saúde: desafios para avaliar a implantação de um "sistema sem muros". Cad Saúde Pública 2004; 20 Suppl 2:S331-6.

10. Mintzberg H. La estructura de las organizaciones. Barcelona: Ariel; 1990.

11. Fratini JRG, Rosita S, Massaroli A. Referência e contra referência: contribuição para a integralidade em saúde. Ciênc Cuid Saúde 2008; 7:65-72.

12. Vázquez ML, Vargas I, Unger JP, De Paepe P, Mogollón-Pérez AS, Samico I, et al. Evaluating the effectiveness of care integration strategies in different health care systems in Latin America: the EQUITY-LA II quasi-experimental study protocol. BMJ Open 2015; 5:e007037.

13. Vargas I, Garcia-Subirats I, Mogollón-Pérez AS, Mendes MFM, Eguiguren P, Cisneros AI, et al. Understanding communication breakdown in the outpatient referral process in Latin America: a cross-sectional study on the use of clinical correspondence in public healthcare networks of six countries. Health Policy Plan 2018; 33:494-504.

14. Vázquez ML, Vargas I, Garcia-Subirats I, Unger JP, De Paepe P, Mogollón-Pérez AS, et al. Doctors' experience of coordination across care levels and associated factors. A crosssectional study in public healthcare networks of six Latin American countries. Soc Sci Med 2017; 182:10-9.

15. Stille CJ, McLaughlin TJ, Primack WA, Mazor KM, Wasserman RC. Determinants and impact of generalist-specialist communication about pediatric outpatient referrals. Pediatrics 2006; 118:1341-9.

16. Vargas I, Mogollón-Pérez AS, De Paepe P, Silva MRF, Unger JP, Vásquez ML. Barriers to health care coordination in market-based and decentralized public health systems: a qualitative study in health care networks of Colombia and Brazil. Health Policy Plan 2016; 31:736-48.

17. Vargas I, Mogollón-Pérez AS, De Paepe P, Silva MRF, Unger JP, Vásquez ML. Do existing mechanisms contribute to improvements in care coordination across level sofcare in health services networks? Opinions of the health personnel in Colombia and Brazil. BMC Health Serv Res 2015; 15:213

18. Vermeir P, Vandijck D, Degroote S, Peleman R, Verhaeghe R, Mortier E, et al. Communication in healthcare: a narrative review of the literature and practical recommendations. Int J Clin Pract 2015; 69:1257-67.

19. Franco CM, Almeida PF, Giovanella L. A integralidade das práticas dos médicos cubanos no Programa Mais Médicos na cidade do Rio de Janeiro, Brasil. Cad Saúde Pública 2018; 34:e00102917.
20. Berendsen AJ, Kuiken A, Benneker WH, MeyboomBJ, Voorn TB, Schuling J. How do general practitioners and specialists value their mutual communication? A survey. BMC Health Serv Res 2009; 9:143.

21. Serra CG. Rodrigues PHA. Avaliação da referência e contrarreferência no Programa Saúde da Família na Região Metropolitana do Rio de Janeiro (RJ, Brasil). Ciênc Saúde Colet 2010; 15 Suppl 3:3579-86.

22. Reig-Mollá B, Bisbal-Andrés E, Sanfélix-Genovés J, Pereiró-Berengner I, Espanza-Pedrol MJ, Martín-González RM. Evaluación de la calidad del documento de interconsulta. ¿Influye la adecuada cumplimentación del médico de família en la respuesta que obtiene del especialista? Aten Primaria 2004; 34:300-5.

23. O’Malley AS, Reschovsky JD. Referral and consultation communication between primary care and specialist physicians: finding common ground. Arch Intern Med 2011; 171:5665.

24. Durbin J, Barnsley J, Finlayson B, Jaakkimainen L, Lin E, Berta W, et al. Quality of communication between primary health care and mental health care: an examination of referral and discharge letters. J Behav Health Serv Res 2012; 39:445-61.

25. Bousquat A, Giovanella L, Campos EMS, Almeida PFD, Martins CL, Mota PHD, et al. Primary health care and the coordination of care in health regions: managers' and users' perspective. Ciênc Saúde Colet 2017; 22:1141-54.

26. Furtado JP. Equipes de referência: arranjo institucional para potencializar a colaboração entre disciplinas e profissões. Interface Comun Saúde Educ 2007; 11:239-55.

27. Martinussen PE. Referral quality and the cooperation between hospital physicians and general practice: the role of physician and primary care factors. Scand J Public Health 2013; 41:874-82.

28. Unnewehr M, Schaaf B, Marev R, Fitch J, Friederichs H. Optimizing the quality of hospital discharge summaries: a systematic review and practical tools. Postgrad Med 2015; 127:630-9.

29. Murphy SF, Lenihan L, Orefuwa F, Colohan G, Hynes I, Collins CG, et al. Electronic discharge summary and prescription: improving communication between hospital and primary care. Ir J Med Sci 2017; 186:455-9.

30. Martínez DH, Navarrete MLV, Lorenzo IV. Factores que influyen en la coordinación entre niveles asistenciales según la opinión de directivos y profesionales sanitarios. Gac Sanit 2009; 23:280-6.

31. Cunha EM, Giovanella L. Longitudinalidade/ continuidade do cuidado: identificando dimensões e variáveis para a avaliação da atenção primária no contexto do sistema público de saúde brasileiro. Ciênc Saúde Colet 2011; 16 Suppl 1:1029-42. 


\section{Abstract}

This article assesses awareness and use of mechanisms for clinical coordination between service levels in two health care networks in the Pernambuco State, Brasil. It is a descriptive, crosssectional, survey-based study. We interviewed 381 doctors from the public primary health care and specialized health care networks in the cities of Caruaru and Recife (Sanitary Districts III and VII). We used a structured questionnaire (COORDENA) in order to assess awareness, frequency and characteristics of the use of the following mechanisms: referral and reply letters, discharge summary, phone and notes (mutual adaptation mechanisms), Health Ministry protocols and joint clinical sessions (standardization mechanisms). We analyzed the data using simple frequencies, means and percentages. In general, primary health care doctors are more familiar with the mechanisms, and use them more frequently, than specialized health care doctors. In the comparison between networks, Recife had better results. Referral and reply letters were the most used (61.4\%) and joint clinical sessions were the least used (8.6\%), in addition to the existence of informal mechanisms (phone 58\%, notes 56.6\%, WhatsApp 2.6\%). Underutilization of mechanisms, divergences in information sent and received between primary health care and specialized health care professionals and inadequate mechanism use suggest communication failures among professionals and service levels. The findings reveal a need for investments that enable awareness, communication and collaboration between professionals, contributing to a better coordination between the different services levels.

Health Care Levels; Comprehensive Health Care; Integrality in Health; Health Evaluation

\section{Resumen}

El artículo evalúa el conocimiento y el uso de los mecanismos de coordinación clínica entre niveles asistenciales en dos redes de atención de salud en el Estado de Pernambuco, Brasil. Se trata de un estudio descriptivo y transversal, basado en una encuesta. Se entrevistaron a 381 médicos de las redes públicas de salud en la atención básica y atención especializada de los municipios de Caruaru y Recife (Distritos Sanitarios III y VII). Se aplicó un cuestionario estructurado (COORDENA) para evaluar el conocimiento, frecuencia y caracteristicas en el uso de los siguientes mecanismos: formularios de referencia y contrarreferencia, resumen de alta, teléfono y notas (mecanismos de adaptación mutua), protocolos del Ministerio de Salud y sesiones clínicas conjuntas (mecanismos de estandarización). Los datos se analizaron mediante frecuencias simples, medias y porcentajes. De manera general, los médicos de la atención primaria conocen y utilizan más los mecanismos que los médicos de la atención especializada. En la comparación entre redes, Recife presentó mejores resultados. Los formularios de referencia y contrarreferencia fueron los más utilizados $(61,4 \%)$ y las sesiones clínicas conjuntas las de menor uso (8,6\%), además de la existencia de mecanismos informales (teléfono un 58\%, nota un 56,6\%, WhatsApp un 2,6\%). La subutilización de los mecanismos, la divergencia de información en el envío y recepción entre los profesionales de la atención primaria y atención especializada y el uso inadecuado de los mismos sugieren fallos en la comunicación entre los profesionales y entre los niveles de atención. Los hallazgos revelan la necesidad de intervenciones que propicien el conocimiento, la comunicación y la colaboración entre profesionales, contribuyendo a una mejor coordinación entre los diferentes niveles asistenciales.

Niveles de Atención de Salud; Atención Integral de Salud; Integralidad en Salud; Evaluación en Salud
Recebido em 16/Ago/2018

Versão final reapresentada em 16/Nov/2018

Aprovado em 28/Jan/2019 